\title{
Fadenlifting und Hyaluronsäurefiller
}

Auch in 2016 veranstaltet die SONEWA Akademie wieder Hands-On-Workshops und Seminare für das Lifting mit VENUS V-Line-PDO-Fäden in Kombination mit Hyaluron-Unterspritzungen mit STYLA$\mathrm{GE}^{\circledast}$. Eine kleine Teilnehmerzahl und hervorragend ausgebildete Trainer garantieren ein schnelles und erfolgreiches Erlernen der neuesten Techniken.

\section{Natürlicher Gesichtsausdruck}

Im Gegensatz zu einem „großen“, operativen Facelift geht es beim Fadenlifting um die Bindegewebsneubildung und den Wiederaufbau des Collagen-Stützgerüstes und nicht darum, das Gewebe in eine neue Position zu ziehen. Der natürliche Gesichtsausdruck bleibt erhalten. Die resorbierbaren Fäden lösen sich nach 6-8 Monaten wieder vollständig auf, das Fadenlifting hält jedoch bis zu 2 Jahre und kann jederzeit problemlos wiederholt werden.
In den Hands-On-Workshops werden den Teilnehmern sowohl die praktische Anwendung der VENUS V-Line-PDOFäden zum Lifting als auch die neuesten Unterspritzungstechniken und volumetrischen Möglichkeiten mit den adaptiven Premium-Fillern von STYLAGE ${ }^{\circledR}$ im Gesicht und in anderen Bereichen des Körpers demonstriert. Nach einer ausführlichen Gesichtsanalyse und Besprechung üben die Teilnehmer dann selbst am Probanden diese kombinierten FaceLift-Techniken, die sich ideal $\mathrm{zu}$ einer modernen, ganzheitlichen Anti-AgingBehandlung ergänzen.

\section{Kosten und Anmeldung}

Die Teilnahmegebühr für den Workshop beträgt $250 €$, die bei einer Bestellung ab $750 €$ innerhalb einer Woche angerechnet werden. In der Workshopgebühr ist das Verbrauchsmaterial während des Hands-

\section{Termine}

Die Mittwochskurse finden von 14:00-18:00 Uhr statt, die Samstagskurse von 10:00-16:00 Uhr.

$\begin{array}{ll}\begin{array}{l}\text { München } \\ \text { Köln }\end{array} & \begin{array}{l}\text { Samstag, 27. Februar } 2016 \\ \text { Mittwoch, 2. März } 2016\end{array} \\ \text { Frankfurt } & \begin{array}{l}\text { Samstag, 12. März } 2016 \\ \text { (Mühlheim-Lämmerspiel) }\end{array} \\ \text { Frankfurt } & \begin{array}{l}\text { Samstag, 9. April 2016 } \\ \text { (Mühlheim-Lämmerspiel) }\end{array} \\ \text { Köln } & \text { Mittwoch, 27. April } 2016 \\ \text { Köln } & \text { Mittwoch, 11. Mai } 2016 \\ \text { München } & \text { Samstag, 11. Juni } 2016\end{array}$

On-Kurses enthalten. Auf Anfrage kann ein eigenes Modell mitgebracht werden. Die Anzahl der Teilnehmer pro Kurs ist begrenzt. Teilnehmer erhalten eine Anmeldebestätigung nach Verfügbarkeit der Plätze. Die Anmeldung erfolgt auf www. sonewa.com oder telefonisch unter 09512960093 oder per Fax an 0951-2960166.

Nach Informationen von SONEWA

\section{Haardiagnostik}

\section{Diagnoseunterstützung durch externes Expertenteam}

Mit FotoFinder Trichovision ${ }^{\circledR}$ sind Ärzte in der Lage, die Ursache von Haarausfall schnell und ohne schmerzhaftes Ausreißen oder Abrasieren von Haaren festzustellen. Mithilfe der Spezialkamera können hochauflösende klinische und

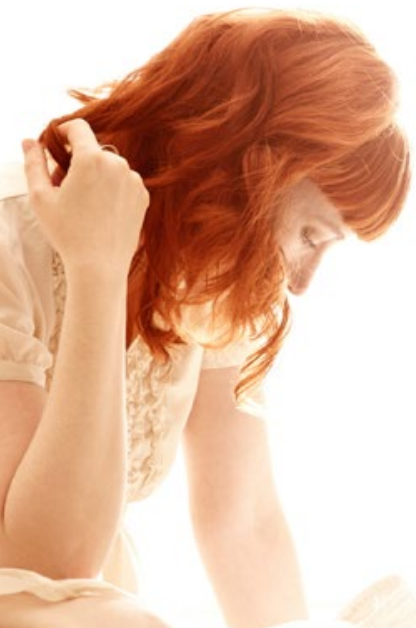

auflichtmikroskopische Bilder der Kopfhaut erstellt werden. Dank der Präzisionsoptik mit optischer Vergrößerung lassen sich die Struktur der Kopfhaut, follikuläre Einheiten und einzelne Haare bis zu 120 -fach vergrößert visualisieren.

\section{Professioneller Patientenservice dank Kooperation}

Per Mausklick können die aufgenommenen Bilder zur Diagnoseunterstützung auf den sicheren Server des TrichoLAB Service, einem exklusiven Partner von FotoFinder, übertragen werden. Im dortigen Labor analysiert ein Expertenteam die Aufnahmen und liefert eine detaillierte trichoskopische Auswertung inklusive Diagnosevorschlag von erfahrenen Ärzten. Das zeitaufwendige Vermessen und Deuten der verschiedenen Parameter lässt sich durch eine Zusammenarbeit mit dem externen Expertenteam komplett outsourcen. So können auch Ärzte ohne umfassende Erfahrung in der Haardiagnostik ihren Patienten einen professionellen Service anbieten.

Darüber hinaus bietet das System umfangreiche Analysefunktionen, die in bestimmten Fällen hilfreich sind, um das genaue Ausmaß des Haarausfalls und den Behandlungsfortschritt zu quantifizieren. Mithilfe der Software FotoFinder Trichoscale pro kann der Arzt zusätzlich selbst verschiedene Parameter des Haarwachstums messen. Dafür werden die Haare auf einer kleinen Fläche an verdeckter Stelle gekürzt. Danach wird ein auflichtmikroskopisches Bild aufgenommen. Sofort berechnet das Programm die Fläche, Haaranzahl und Haardichte sowie Anzahl und Dichte der Vellus- und Terminalhaare. Zudem werden die durchschnittliche und kumulative Haardicke sowie die follikulären Einheiten gemessen. Nach 48 Stunden lässt sich im Trichogramm-Modus die Anagen-Telogen-Rate bestimmen und für jedes einzelne Haar die Länge aufzeigen. So wird auf einen Blick sichtbar, welche Haare nach dem Kürzen gewachsen sind und welche nicht.

Nach Informationen von FotoFinder Systems 